\title{
Estimulación de la atención sostenida en el neonato previo al tamizaje auditivo, reducción de falsos positivos
}

\author{
Stimulation of sustained attention in the neonate prior to \\ auditive screening, reduction of false positive
}

\author{
Daniel Alfredo Alcivar Arteaga, * Alberto Orozco Gutiérrez*
}

\section{Resumen}

Las pruebas de emisión otoacústicas y la respuesta auditiva del tallo cerebral se utilizan como herramientas de detección para el tamizaje auditivo en recién nacidos. Sin embargo, se presentan elevadas tasas de falsos positivos, por lo que se determinó si la estimulación auditiva previa al tamizaje disminuye la incidencia de falsos positivos. Material y métodos: Estudio de casos y controles, longitudinal y experimental; al grupo de casos se le dio un estímulo auditivo previo al tamizaje auditivo con la Sonata No. 6 en Re Mayor de Mozart de 50 a 320 kbps. Resultados: Ambos grupos de estudio fueron estadísticamente iguales; se observó que la edad gestacional no tiene relación con los resultados del tamizaje $(p=0.782)$. Existe diferencia significativa entre los resultados obtenidos en el tamizaje con y sin estimulación auditiva previa $(p=0.017)$. Se evidencia mayor especificidad en el uso de estimulación auditiva previa que la realización del tamizaje sin dicho estímulo (93.75 vs. 84.49\%). Conclusión: La estimulación auditiva disminuye el número de falsos positivos, lo que posiblemente se asocia a que se aísla al menor del ruido ambiental y se favorece el estímulo de la atención sostenida al no poder solicitar el uso de la red organizativa y ejecutiva de la atención.

Palabras clave: Emisiones otoacústicas, hipoacusia, estimulación auditiva, recién nacidos.

\footnotetext{
* Departamento de Neonatología. Hospital Ángeles Pedregal. Facultad Mexicana de Medicina Universidad La Salle.
}

Correspondencia:

Daniel Alcivar Arteaga

Correo electrónico: dalcivar.md@gmail.com

Aceptado: 09-09-2019.

www.medigraphic.com/actamedica

\section{Abstract}

Otoacoustic emission tests (OAE) and auditory response of the brain stem are used as screening tools for hearing screening in newborns. However, there are high rates of false positives. Therefore, it is determined whether the auditory stimulation prior to screening affected the incidence of false positives. Material and methods: Case and control study, longitudinal and experimental; the case group was given an auditory stimulus prior to auditory screening with Mozart Sonata No. 6 in D at 50 to 320 kbps. Results: Both study groups were statistically equal; It was found that gestational age is not related to screening results $(p=0.782)$. There is a significant difference between the results obtained in the screening with and without previous auditory stimulation $(p=0.017)$. Showing a greater specificity in the use of prior auditory stimulation than screening without such stimulation ( 93.75 vs. $84.49 \%$ ). Conclusion: The auditory stimulation modified the number of false positives possibly associated with the lower level of environmental noise and the stimulation of sustained attention is favored by not being able to request the use of the organizational and executive network of care.

Keywords: Otoacoustic emissions, hearing loss, auditory stimulation, newborns.

\section{INTRODUCCIÓN}

La pérdida de audición en los niños se reconoce como un trastorno común, ${ }^{1}$ la prevalencia de pérdida auditiva congénita permanente en recién nacidos sin riesgo es de aproximadamente uno en 1,000 para la hipoacusia bilateral profunda, y de 3-4 por 1,000 para casos unilaterales o leves. ${ }^{2-5}$ Investigaciones han demostrado que si esta pérdida no se detecta de manera oportuna, se corre el riesgo de retrasos en la formación del habla, el lenguaje y más retrasos globales en el desarrollo cognitivo y social. ${ }^{6}$ 
El objetivo de la evaluación auditiva universal del recién nacido (UNHS) es la detección temprana de la pérdida auditiva y la intervención inmediata a través de un enfoque integrado, interdisciplinario y centrado en la familia. Ésta busca evaluar a todos los bebés entre el primer mes de vida, diagnosticar de manera concluyente la pérdida auditiva congénita permanente antes del tercer mes y tratar al paciente antes del sexto mes de vida. ${ }^{7}$

Estudios previos han demostrado que los beneficios de un programa de evaluación auditiva universal en recién nacidos superan los inconvenientes y los costos asociados. ${ }^{8}$ El diagnóstico precoz ahorra los costos de la intervención intensiva del habla y del lenguaje y los servicios educativos especiales. . $^{9-12}$

Actualmente, las pruebas de emisión otoacústicas (OAE) y/o respuesta auditiva del tallo cerebral (ABR) se utilizan como herramientas de detección en programas de tamizaje auditivo en recién nacidos. La prueba OAE es rápida, fácil de realizar y asequible, convirtiéndola en una excelente herramienta a utilizar en los programas de tamiz auditivo en recién nacidos. ${ }^{13}$

Para atender y dar solución a este problema de salud pública en el país, la SSA diseñó el Programa de Tamiz Auditivo Neonatal e Intervención Temprana, avalado por la norma oficial mexicana: NOM-034-SSA2-2013 para la prevención y control de defectos al nacimiento. ${ }^{14}$ Esta misma recomendación ha sido emitida por los institutos nacionales de salud de EUA, en consenso con la Academia Americana de Pediatría. 6,15

Sin embargo, las pruebas de OAE se han asociado con tasas de falsos positivos (1.9\%) cuando éstas son utilizadas como prueba de tamizaje en recién nacidos. ${ }^{16-18}$

Estos resultados falsos positivos se han asociado con condiciones transitorias propias del conducto auditivo externo como del oído medio del recién nacido (por ejemplo: colapso del conducto auditivo externo, presencia de detritus de vérmix, presencia de líquido amniótico y mesénquima en oído medio), ${ }^{19-21}$ así como niveles de ruido ambiental. ${ }^{22,23}$

Estas altas tasas de falsos positivos son motivo de importante preocupación debido a que se puede poner en duda el programa de tamizaje, además de llevar a un motivo de interconsulta o utilización de estudios diagnósticos de manera innecesaria. ${ }^{9,24}$ Asimismo, estos resultados conducen a un estado de ansiedad familiar cambiante. ${ }^{17,25-27}$

Razón por la cual se propone mediante este estudio determinar la incidencia de falsos positivos en el tamizaje auditivo en los neonatos atendidos en el Hospital Ángeles Pedregal y valorar un mecanismo de estimulación auditiva previa a la realización del tamizaje para reducir los falsos positivos.

\section{MATERIAL Y MÉTODOS}

Se realizó un estudio de casos y controles, longitudinal y experimental en la población de neonatos que nacen en el Hospital Ángeles Pedregal. Dicho estudio se realizó entre enero y diciembre de 2017, considerando que para un estudio con un margen de error de $5 \%$ se requería al menos 59 pacientes para cada grupo de estudio según lo determinado con el programa Epilnfo v7.2.2 (USA). Los pacientes incluidos en el estudio fueron neonatos nacidos en el Hospital Ángeles Pedregal que hayan estado en cunero fisiológico o Unidad de Cuidados Intermedios Neonatales y cuyo médico tratante haya indicado la prueba de tamizaje auditivo.

Los pacientes fueron aleatorizados de manera simple; al grupo control se le realizó el tamizaje auditivo previo a su egreso y al grupo de casos se le aplicó una estimulación auditiva previa al tamizaje auditivo empleando el equipo Otodynamics Otoport DP+TE (SN DTC/14128632) previo consentimiento de los padres o tutores. La estimulación auditiva se dio durante 10 minutos antes de realizar el tamizaje por medio de audífonos (Figuras 1 A y B) empelando auriculares marca iPhone adaptados a auriculares flexibles desechables marca Natus ${ }^{\circledR}$; el estímulo se dio con música compuesta por Mozart, se empleó la Sonata $N^{0} 6$ en Re Mayor a un volumen de $50 \mathrm{~dB}$ (Figura 1 A y B) utilizando para la reproducción la aplicación de Spotify para iPhone (Apple, USA) versión 8.4.51.696 con una calidad de audio de 320 kbps. Para la medición de los decibeles se utilizó la aplicación Decibel X PRO versión 6.2 .3 (2974) para iPhone (Apple, USA).

Tanto para casos como controles, cuando se obtenía un resultado anormal o «No pasa» se realizó una segunda evaluación a las tres a cuatro semanas; en los pacientes en quienes se realizó un segundo tamizaje ya no se empleó una estimulación auditiva previa a la valoración. El análisis estadístico se llevó acabo con el programa IBM SPSS v25 (USA), considerando significativo el resultado cuando el valor de $\mathrm{p}$ sea $<0.05$.

\section{RESULTADOS}

El estudio se compuso de 489 pacientes en total, de los cuales 61 fueron casos y el resto controles. De la muestra total $53.4 \%$ de los casos corresponden a hombres y $43.6 \%$ a mujeres. Las características generales de ambos grupos se resumen en la Tabla 1.

Derivado de que se encontró una diferencia significativa entre ambos grupos en relación a las semanas de gestación a las que se dio el nacimiento, se realizó una prueba de correlación por $\mathrm{R}$ de Pearson entre dicha variable y el resultado del primer tamizaje auditivo; el resultado indicó que no se encontraba una correlación significativa ( $p=$ 0.782) (Tabla 2).

Posteriormente se calculó la especificidad y el valor predictivo positivo para el tamizaje auditivo con y sin estí- 
Figura 1:

A) Audífonos desechables adaptados para uso en neonato. B) Evaluación de $\mathrm{dB}$ del estímulo auditivo con la aplicación Decibel X PRO versión 6.2 .3 (2974) para iPhone (Apple, USA).

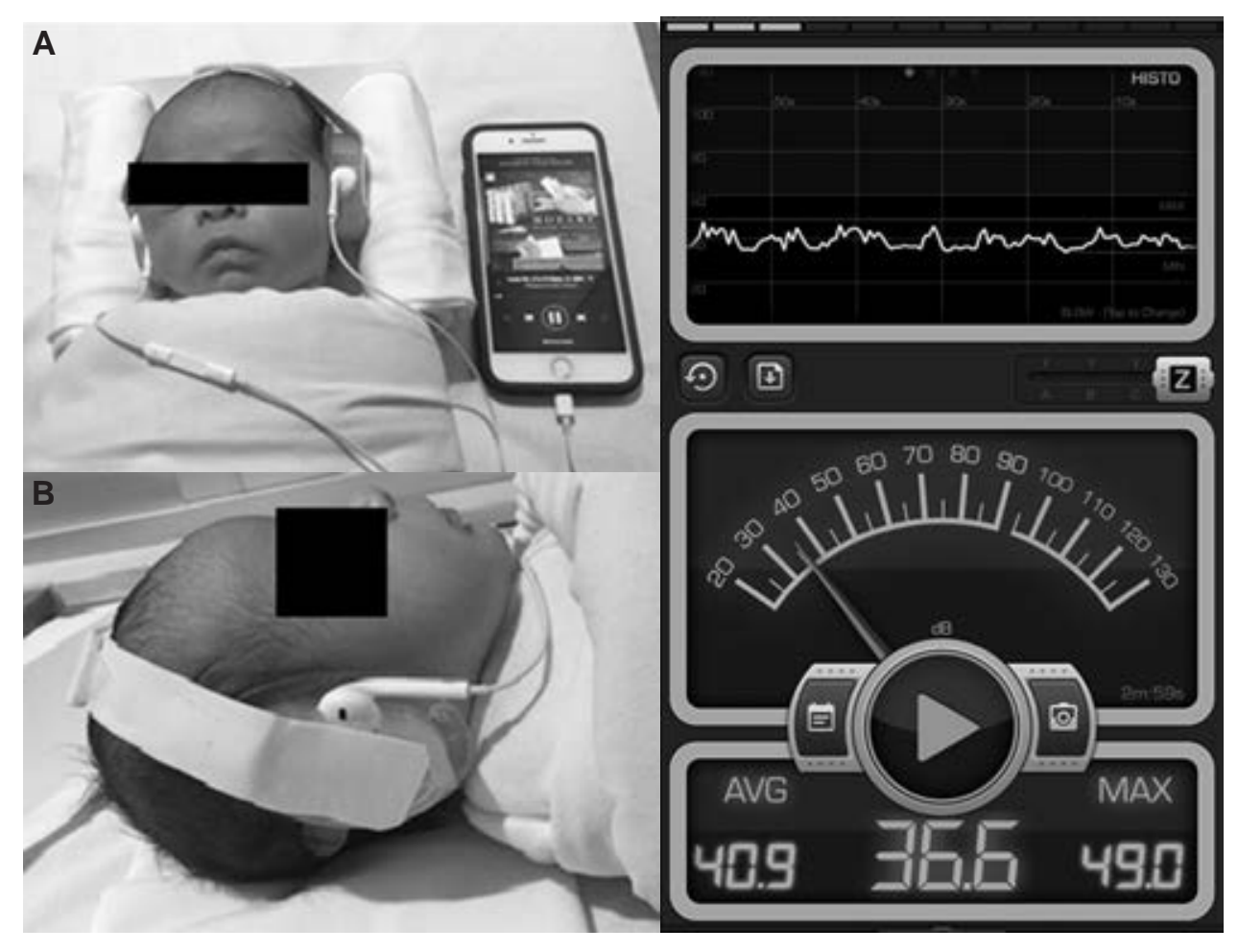

mulo previo (Tabla 3). No se realizó cálculo de sensibilidad debido a que a los pacientes que no presentaron datos anómalos en el primer tamizaje se desconoce si a posteriori presenten déficit auditivo.

\section{DISCUSIÓN}

Como se muestra en nuestro estudio la población de recién nacidos presenta mayor porcentaje de población masculina que femenina, siendo que los reportes del Instituto Nacional de Estadística y Geografía señalan que en nuestro país la tasa de nacimiento de hombres y mujeres es casi idéntica desde el año 2015. ${ }^{28}$

Si bien se encontró diferencia significativa entre los casos y los controles en relación a la edad gestacional, se demostró que la edad gestacional no es un factor que guarde correlación con los resultados obtenidos en el tamizaje auditivo de nuestro estudio; razón por la cual dicha variable fue descartada como mecanismo de explicación para los resultados obtenidos en el tamizaje auditivo llevado a cabo en el presente estudio, es decir, que las diferencias en la microestructura de la materia blanca que se da entre los neonatos a término y los pretérmino no afectan la valoración de las vías auditivas. ${ }^{29}$

Sin embargo, cabe resaltar que la edad gestacional del grupo de casos apenas alcanza el ideal para una edad gestacional apropiada, ${ }^{30}$ lo cual es un punto interesante a estudiar más adelante en la población de dicho centro de atención médica.

De manera interesante el peso gestacional de ambos grupos se encontró dentro de los recomendados como nacimiento a término. ${ }^{30}$ Adicionalmente, el tamizaje auditivo se realizó a una edad acorde a lo señalado en la Norma Oficial Mexicana para la prevención y control de defectos al nacimiento y lo señalado como adecuado para recién nacidos. ${ }^{14}$

En relación a la incidencia de casos de tamizaje alterado en los controles, en nuestro estudio se encontró mayor número de casos positivos a alteración en comparación con lo reportado en un estudio con una población similar a la nuestra en Chile. ${ }^{31}$ En cuanto a la incidencia de fallo auditivo en el neonato después del segundo escrutinio, este resultado es ligeramente superior a la incidencia que se ha reportado a nivel mundial que es de uno a tres casos por cada 1,000 recién nacidos. ${ }^{7}$ Además, no se encontró diferencia entre los resultados entre la presencia de factores de riesgo asociados a alteraciones en la audición en ambos grupos, por lo que dichos factores no interfieren en los resultados obtenidos en el presente estudio. ${ }^{32}$

Considerando lo anterior podemos inferir que la diferencia entre la cantidad de falsos positivos que se dieron en el tamizaje auditivo con y sin estimulación auditiva previa se deben directamente al hecho del estímulo empleado.

Sin embargo, tanto el método automático como las emisiones otoacústicas siguen presentando una alta tasa de 
pacientes que deben someterse a una segunda prueba, es decir, un alto grado de falsos positivos como se observó en nuestro estudio. Esto en contraste con lo que se recomienda o espera, que debe ser de $4 \%$ o menos. ${ }^{33}$

\begin{tabular}{|c|c|c|c|}
\hline & $\begin{array}{c}\text { Controles } \\
n=428(\%)\end{array}$ & $\begin{array}{c}\text { Casos } \\
n=61(\%)\end{array}$ & $\mathrm{p}$ \\
\hline Sexo & & & 0.127 \\
\hline Hombres & $234(54.7)$ & $27(44.3)$ & \\
\hline Mujeres & 194 (45.3) & $34(55.7)$ & \\
\hline $\mathrm{SDG}, \mathrm{m} \pm \mathrm{DE}$ & $37.62 \pm 1.589$ & $38.13 \pm 1.033$ & 0.0158 \\
\hline $\begin{array}{l}\text { Peso al nacimiento } \\
(\mathrm{kg}), \mathrm{m} \pm \mathrm{DE}\end{array}$ & $2,869 \pm 470.7$ & $2,949 \pm 484.6$ & 0.2158 \\
\hline $\begin{array}{l}\text { Días de estancia } \\
\text { hospitalaria, } m \pm D E\end{array}$ & $3.294 \pm 1.113$ & $3.656 \pm 3.119$ & 0.0813 \\
\hline $\begin{array}{l}\text { Edad al tamizaje } \\
\text { (días), } m \pm \mathrm{DE}\end{array}$ & $3.643 \pm 5.31$ & $2.541 \pm 0.6474$ & 0.1071 \\
\hline Nacimiento & & & 0.239 \\
\hline Parto & $83(19.4)$ & 8 (13.1) & \\
\hline Cesárea & $345(80.6)$ & $53(86.9)$ & \\
\hline $\begin{array}{l}\text { Destino, } \\
\text { postnacimiento }\end{array}$ & & & 0.729 \\
\hline Cunero & $387(90.4)$ & $56(91.8)$ & \\
\hline UCIN & 41 (91.8) & $5(8.2)$ & \\
\hline Ventilación mecánica & & & 0.884 \\
\hline Sí & $6(1.4)$ & $1(1.6)$ & \\
\hline No & $422(98.6)$ & $60(98.4)$ & \\
\hline Oxígeno & & & 0.320 \\
\hline Sí & $37(8.6)$ & $3(4.9)$ & \\
\hline No & 391 (91.4) & $58(95.1)$ & \\
\hline $\begin{array}{l}\text { Antibiótico al } \\
\text { nacimiento }\end{array}$ & & & 0.444 \\
\hline Sí & $15(3.5)$ & $1(1.6)$ & \\
\hline No & $413(96.5)$ & $60(98.4)$ & \\
\hline Diurético & & & 0.396 \\
\hline Sí & $5(1.2)$ & $0(0.0)$ & \\
\hline No & $423(98.8)$ & $61(100.0)$ & \\
\hline Hiperbilirrubinemia & & & 0.656 \\
\hline Sí & $16(3.7)$ & $3(4.9)$ & \\
\hline No & 412 (96.3) & $58(95.1)$ & \\
\hline $\begin{array}{l}\text { Antecedentes fami- } \\
\text { liares de problemas } \\
\text { auditivos }\end{array}$ & & & $\mathrm{NC}$ \\
\hline No & $428(100.0)$ & $61(100.0)$ & \\
\hline Enfermedad materna & & & 0.705 \\
\hline Sí & $1(0.2)$ & $0(0.0)$ & \\
\hline No & $427(99.8)$ & 61 (100.0) & \\
\hline
\end{tabular}

El mecanismo por el cual la estimulación auditiva previa redujo la cantidad de falsos positivos puede darse por algunas situaciones. Partamos del hecho de que los audífonos nos permiten aislar al neonato del ruido del ambiente; sin embargo, dicha situación se logra actualmente con el empleo de mecanismos automatizados. ${ }^{34}$ De esta manera el procedimiento de aislar el sonido para enfocar la atención en el sonido que se emplea, se descarta como mecanismo por el cual se reducen los falsos positivos en el uso de una estimulación previa al tamizaje.

Lo anterior nos lleva al tema de la atención, la cual se ha considerado como una variable a considerar aun en el estudio de audiometría en los adultos. ${ }^{35}$ Siendo que en el caso de la valoración auditiva se requiere un estado mayor que el estado arousal, se requiere una atención focal, sostenida y selectiva. ${ }^{36}$ Sin embargo, no existe aún un nivel de lenguaje y comunicación con el menor como se da con un niño más grande o un adulto, por lo que es posible que la estimulación continua previa al tamizaje favorece al neonato a presentar una atención sostenida y selectiva a un único estímulo que sea el sonido. Esto se ha asociado en el estudio de la respuesta y reacciones temperamentales hacia objetos físicos. ${ }^{37}$

Biológicamente esto es justificable, ya que desde el nacimiento el nervio vestibulococlear ya está mielinizado y es funcional, al igual que la regulación de la atención y un estado mínimo de conciencia, aun cuando no se tenga completo dominio sobre él y por ello se requiera estimularlo para favorecer su reacción. ${ }^{38}$ Por lo cual podemos afirmar que la estimulación previa al tamizaje auditivo es

\begin{tabular}{|c|c|c|c|}
\hline & $\begin{array}{c}\text { Controles } \\
n=428(\%)\end{array}$ & $\begin{array}{c}\text { Casos } \\
n=61(\%)\end{array}$ & $p$ \\
\hline Primer tamizaje auditivo & & & 0.017 \\
\hline Normal & 347 (81.07) & $57(93.44)$ & \\
\hline No pasa & 81 (18.92) & $4(6.55)$ & \\
\hline Oído lesionado & & & 0.063 \\
\hline Derecho & 70 (86.42) & $2(50.00)$ & \\
\hline Izquierdo & $6(7.4)$ & $1(25.00)$ & \\
\hline Ambos & $5(6.17)$ & $1(25.00)$ & \\
\hline Segundo tamizaje auditivo & & & 0.06 \\
\hline Normal & 78 (96.3) & $3(75.00)$ & \\
\hline No pasa & $3(3.7)$ & $1(1.00)$ & \\
\hline Oído lesionado & & & 0.014 \\
\hline Derecho & $2(66.7)$ & $1(100.00)$ & \\
\hline Izquierdo & $1(33.3)$ & 0 & \\
\hline
\end{tabular}


Tabla 3: Especificidad y valor predictivo positivo del primer tamizaje en ambos grupos.

\begin{tabular}{lcc} 
& $\begin{array}{c}\text { Sin estímulo } \\
\text { previo (\%) }\end{array}$ & $\begin{array}{c}\text { Con estímulo } \\
\text { previo (\%) }\end{array}$ \\
\hline Especificidad & 84.49 & 93.75 \\
Valor predictivo positivo & 3.70 & 25.00 \\
\hline
\end{tabular}

importante para favorecer diferentes niveles de atención y mejorar su atención al estudio de tamizaje; eliminando de esta manera todas las reacciones que tenía el recién nacido previo al tamizaje y se acostumbre y adecue a un objeto en sus pabellones auditivos.

Adicionalmente, con el uso del estímulo auditivo previo al tamizaje se logra una especificidad acorde a lo esperado en la prueba empleada y similar a lo esperado en los métodos automatizados. ${ }^{39}$ Incluso mejora los resultados en especificidad y reducción de falsos positivos, aun cuando se haga un estudio de comparación entre personas con diferente tiempo de entrenamiento en la aplicación de la prueba. ${ }^{40}$ Por lo que la modificación propuesta a la técnica es totalmente beneficiosa para el estudio y la reducción de falsos positivos.

\section{CONCLUSIÓN}

La estimulación auditiva disminuye el número de falsos positivos, lo que posiblemente se asocia a que se aísla al menor del ruido ambiental y se favorece el estímulo de la atención sostenida al no poder solicitar el uso de la red organizativa y ejecutiva de la atención.

\section{REFERENCIAS}

1. Kerschner JE. Neonatal hearing screening: to do or not to do. Pediatric Clinics of North America. 2004; 51 (3): 725-736, x.

2. Finitzo $\mathrm{T}$, Albright $\mathrm{K}, \mathrm{O}^{\prime} \mathrm{Neal} J$. The newborn with hearing loss: detection in the nursery. Pediatrics. 1998; 102 (6): 1452-1460.

3. Prieve BA, Stevens F. The New York State universal newborn hearing screening demonstration project: introduction and overview. Ear Hear. 2000; 21 (2): 85-91.

4. Mehl AL, Thomson V. The Colorado newborn hearing screening project, 1992-1999: on the threshold of effective population-based universal newborn hearing screening. Pediatrics. 2002; 109 (1): e7.

5. Ur Rehman M, Mando K, Rahmani A, Imran A, Ur Rehman N, Gowda $K$ et al. Screening for neonatal hearing loss in the Eastern region of United Arab Emirates. East Mediterr Health J. 2012; 18 (12): 12541256.

6. American Academy of Pediatrics JColH. Year 2007 position statement: Principles and guidelines for early hearing detection and intervention programs. Pediatrics. 2007; 120 (4): 898-921.

7. Erenberg A, Lemons J, Sia C, Trunkel D, Ziring P. Newborn and infant hearing loss: detection and intervention. American Academy of
Pediatrics. Task Force on Newborn and Infant Hearing, 1998-1999. Pediatrics. 1999; 103 (2): 527-530.

8. Wolff R, Hommerich J, Riemsma R, Antes G, Lange S, Kleijnen J. Hearing screening in newborns: systematic review of accuracy, effectiveness, and effects of interventions after screening. Arch Dis Child. 2010; 95 (2): 130-135.

9. Nelson HD, Bougatsos C, Nygren P, Force USPST. Universal newborn hearing screening: systematic review to update the 2001 US Preventive Services Task Force Recommendation. Pediatrics. 2008; 122 (1): e266-276.

10. Thompson DC, McPhillips H, Davis RL, Lieu TA, Homer CJ, Helfand $M$. Universal newborn hearing screening: summary of evidence. JAMA. 2001; 286 (16): 2000-2010.

11. Gorga MP, Neely ST. Cost-effectiveness and test-performance factors in relation to universal newborn hearing screening. Mental retardation developmental disabilities research reviews. 2003; 9 (2): 103-108.

12. Porter HL, Neely ST, Gorga MP. Using benefit-cost ratio to select Universal Newborn Hearing Screening test criteria. Ear Hearing. 2009; 30 (4): 447.

13. Kennedy CR, Kimm L, Dees DC, Evans PI, Hunter M, Lenton S et al. Otoacoustic emissions and auditory brainstem responses in the newborn. Arch Dis Child. 1991; 66 (10 Spec No): 1124-1129.

14. Unions HCdl. Norma Oficial Mexicana Para la prevención y control de defectos al nacimiento. 2014.

15. Erenberg A, Lemons J, Sia C, Trunkel D, Ziring P. Task Force on Newborn and Infant Hearing. Newborn and infant hearing loss: detection and intervention. Pediatrics. 1999; 103 (2): 527-530.

16. Boone RT, Bower CM, Martin PF. Failed newborn hearing screens as presentation for otitis media with effusion in the newborn population. Int J Pediatr Otorhinolaryngol. 2005; 69 (3): 393-397.

17. Clemens CJ, Davis SA, Bailey AR. The false-positive in universal newborn hearing screening. Pediatrics. 2000; 106 (1): E7.

18. Clemens CJ, Davis SA. Minimizing false-positives in universal newborn hearing screening: a simple solution. Pediatrics. 2001; 107 (3): e29-e.

19. Chang KW, Vohr BR, Norton SJ, Lekas MD. External and middle ear status related to evoked otoacoustic emission in neonates. Arch Otolaryngol Head Neck Surg. 1993; 119 (3): 276-282.

20. Thornton AR, Kimm L, Kennedy C, Cafarelli-Dees D. Externaland middle-ear factors affecting evoked otoacoustic emissions in neonates. Br J Audiol. 1993; 27 (5): 319-327.

21. Couto CMd, Carvallo RMM. The effect external and middle ears have in otoacoustic emissions. Rev Bras Otorrinolaringol. 2009; 75 (1): 15-23.

22. Jacobson JT, Jacobson CA. The effects of noise in transient EOAE newborn hearing screening. Int J Pediatr Otorhinolaryngol. 1994; 29 (3): 235-248.

23. Headley GM, Campbell DE, Gravel JS. Effect of neonatal test environment on recording transient-evoked otoacoustic emissions. Pediatrics. 2000; 105 (6): 1279-1285.

24. Stein LK. Factors influencing the efficacy of universal newborn hearing screening. Pediatr Clin North Am. 1999; 46 (1): 95-105.

25. Young A, Andrews E. Parents' experience of universal neonatal hearing screening: a critical review of the literature and its implications for the implementation of new UNHS programs. J Deaf Stud Deaf Educ. 2001; 6 (3): 149-160.

26. Van der Ploeg CP, Uilenburg NN, Kauffman-de Boer MA, OudesluysMurphy AM, Verkerk PH. Newborn hearing screening in youth health care in the Netherlands: National results of implementation and follow-up. Int J Audiol. 2012; 51 (8): 584-590.

27. Fox R, Minchom S. Parental experiences of the newborn hearing screening programme in Wales: a postal questionnaire survey. Health Expect. 2008; 11 (4): 376-383.

28. INEGI. Población. https://www.inegi.org.mx/temas/estructura/2015.

29. Ou X, Glasier CM, Ramakrishnaiah RH, Kanfi A, Rowell AC, Pivik RT et al. Gestational age at birth and brain white matter development in term-born infants and children. AJNR Am J Neuroradiol. 2017; 38 (12): 2373-2379. 
30. Malin G, Morris R, Riley R, Teune M, Khan K. When is birthweight at term ( $\geq 37$ weeks' gestation) abnormally low? A systematic review and meta-analysis of the prognostic and predictive ability of current birthweight standards for childhood and adult outcomes. BJOG. 2015; 122 (5): 634-642.

31. Nazar MG, Goycoolea VM, Godoy SJM, Ried GE, Sierra GM Evaluación auditiva neonatal universal: revisión de 10.000 pacientes estudiados. Rev Otorrinolaringol Cir Cabeza Cuello. 2009; 69: 93-102.

32. Coello F, Cuevas H, Andrade E. El tamizaje auditivo neonatal en Ecuador, un compromiso ineludible. Revista de la Facultad de Ciencias Médicas. 2016; 41 (1): 177-184.

33. Wroblewska-Seniuk KE, Dabrowski P, Szyfter W, Mazela J. Universal newborn hearing screening: methods and results, obstacles, and benefits. Pediatr Res. 2017; 81 (3): 415-422.

34. Schönweiler R, Schmidt C-M. Universal auditory screening of neonates and hearing disorders in childhood. Dtsch Arztebl Int. 2009; 106 (20): 355-356.
35. Istre JC, LeJeune JF. Variables in objective audiometry. Southern Medical Journal. 1975; 68 (7): 844-848.

36. Lubrini G, Periáñez J, Ríos-Lago. Estimulación cognitiva y rehabilitación neuropsicológica de la atención. Neuropsicol. 2009.

37. Pérez-López J, Herrero ÁD, Meca JS. Relaciones entre irritabilidad neonatal y reacciones temperamentales hacia objetos físicos. Anuario de psicología/The UB. Journal of Psychology. 1999; 30 (3): 59-70.

38. Pérez HE. Desarrollo de los procesos atencionales. Universidad Complutense de Madrid, Servicio de Publicaciones; 2008.

39. Eiserman WD, Hartel DM, Shisler L, Buhrmann J, White KR, Foust T. Using otoacoustic emissions to screen for hearing loss in early childhood care settings. Int J Pediatr Otorhinolaryngol. 2008; 72 (4): 475-482.

40. Ricalde RR, Chiong CM, Labra PJP. Current assessment of newborn hearing screening protocols. Curr Opin Otolaryngol Head Neck Surg. 2017; 25 (5): 370-377. 